05,11

\title{
Влияние структурных дефектов на магнитные свойства монокристалла $\mathrm{EuBaCo}_{1.90} \mathrm{O}_{5.36}$
}

\author{
() Т.И. Арбузова, С.В. Наумов , С.В. Телегин
}

Институт фризики металлов им. М.Н. Михеева УрО РАН,

Екатеринбург, Россия

ฯ E-mail: naumov@imp.uran.ru

(Поступила в Редакцию 17 мая 2017 г.)

Исследовано влияние структурных дефектов в кобальтовых и кислородных подрешетках при сохранении средней степени окисления $3+$ всех ионов кобальта на магнитные свойства монокристалла $\mathrm{EuBaCo}_{1.90} \mathrm{O}_{5.36}$. Проведено сравнение магнитных свойств монокристалла и поликристаллического образца соответствующего состава в области $T=200-650 \mathrm{~K}$. Полученные данные показали, что в кобальт-дефицитных образцах $\mathrm{EuBaCo}_{2-x} \mathrm{O}_{5.5-\delta}$ реализуется трехмерное $X Y$ ферримагнитное упорядочение магнитных подрешеток. Значения эффективного магнитного момента в области $T>480 \mathrm{~K}$ указывают на присутствие $I S$ - и $H S$-состояний ионов $\mathrm{Co}^{3+}$. Большое различие значений $\mu_{\text {eff }}$ для монокристалла и поликристалла $\mathrm{EuBaCo}_{1.90} \mathrm{O}_{5.36}$ может быть связано с тем, что спины магнитных ионов лежат в плоскости $a b$. Магнитное поле, направленное вдоль плоскости $a b$, сильно влияет на магнитное упорядочение в области $T<300 \mathrm{~K}$.

Работа выполнена в рамках государственного задания ФАНО России (тема „Спин“, № 01201463330) при частичной поддержке программы УрО РАН (проект № 15-9-2-4).

DOI: 10.21883/FTT.2018.01.45292.152

\section{1. Введение}

Двойные слоистые кобальтиты $L n \mathrm{BaCo}_{2} \mathrm{O}_{5.50 \pm \delta}(\mathrm{Ln}-$ редкоземельный элемент, $0 \leq \delta \leq 0.5$ ) относятся к классу магнитных полупроводников, в которых сильная взаимосвязь между магнитной, электронной и структурной подсистемами приводит к ряду необычных свойств. В частности, наблюдаются магнитные переходы из антиферромагнитного (AF) в ферромагнитное (FM) состояние и при повышении температуры в парамагнитное состояние $[1,2]$. В области $T=300-400 \mathrm{~K}$ слоистые кобальтиты испытывают переход изолятор-металл (I/M), а при низких температурах $T<125 \mathrm{~K}$ показывают эффект магнитосопротивления (MR). B EuBaCo $2 \mathrm{O}_{5.4} \mathrm{MR}$ эффект составляет $20 \%$, а в $\mathrm{GdBaCo}_{2} \mathrm{O}_{5.4}-22 \%$ [3]. $\mathrm{B}$ то же время эти соединения являются перспективным материалом для практического применения, например для катодов твердотопливных элементов.

Система $L n \mathrm{BaCo}_{2} \mathrm{O}_{5.50 \pm \delta}$ имеет непрерывный ряд твердых растворов, в которых содержание кислорода может изменяться от 5 до 6. При этом ионы кобальта могут иметь валентное состояние $2+, 3+$ и $4+$. В составе $L n \mathrm{BaCo}_{2} \mathrm{O}_{5}$ ионы Cо находятся в пирамидальном окружении, а в $\operatorname{Ln} \mathrm{BaCo}_{2} \mathrm{O}_{6}$ - в октаэдрическом окружении. В $L n \mathrm{BaCo}_{2} \mathrm{O}_{5.50}$ средняя степень окисления ионов кобальта - 3+. В перовскитной структуре расщепление $3 d$ уровней переходных элементов кристаллическим полем и внутриатомный обмен имеют близкие значения, поэтому в зависимости от объема элементарной ячейки и температуры ионы $\mathrm{Co}^{3+}$ могут находиться в высокоспиновом HS-состоянии $(S=2)$, в промежуточном (IS) c $S=1$ и низкоспиновом (LS) немагнитном состояниях с $S=0 \quad[4,5]$. В структуре перовскита IS-состояния предпочтительны для пирамидального окружения кислорода, а для октаэдрического окружения - LS-состояния. Общепринятым считается, что при низких температурах в октаэдрических позициях расположены в шахматном порядке 0.5 (LS) и 0.5 (HS) состояний ионов $\mathrm{Co}^{3+}$, a в пирамидах - IS-состояния [6]. Экспериментальные данные по дифракции рентгеновских лучей [7] и данные по ядерному магнитному резонансу (ЯМР) [8] подтверждают такое распределение $\mathrm{Co}^{3+}$ ионов.

Магнитные измерения монокристалла $\mathrm{GdBaCo}_{2} \mathrm{O}_{5.50}$ показали, что в интервале температур 200-300 K присутствуют FM-области, внедренные в АF-матрицу, т.е. магнитное состояние является неоднородным [1]. В этой температурной области обнаружена также большая анизотропия намагниченности, что, вероятно, указывает на упорядочение $\mathrm{Co}^{3+}$ спинов в плоскости $a b . \mathrm{B} L n \mathrm{BaCo}_{2} \mathrm{O}_{5.50}$ ниже температуры перехода металл-изолятор $T(I / M) \approx 360 \mathrm{~K}$ антиферромагнитный порядок матрицы может обеспечить сверхобмен $\mathrm{Co}^{3+}(\mathrm{IS})-\mathrm{O}-\mathrm{Co}^{3+}(\mathrm{IS})$ и $\mathrm{Co}^{3+}(\mathrm{HS})-\mathrm{O}-\mathrm{Co}^{3+}(\mathrm{HS})$. Ферромагнитный порядок может быть связан со сверхобменом $\mathrm{Co}^{3+}(\mathrm{HS})-\mathrm{O}-\mathrm{Co}^{3+}(\mathrm{IS})$ между ближайшими соседями [4]. Согласно симметрийному рассмотрению слоистых кобальтитов, конкуренция между AF и FM обменными взаимодействиями может привести к ферримагнитной структуре [6]. При этом не исключена неколлинеарная магнитная структура [9].

Основное внимание в литературе уделялось изучению влияния содержания кислорода на магнитные свойства слоистых кобальтитов $L n \mathrm{BaCo}_{2} \mathrm{O}_{5.50 \pm \delta}$. В данной работе поставлена задача изучить влияние структурных дефектов в кобальтовых и кислородных подрешетках при сохранении валентности 3+ всех ионов кобальта на 
магнитные свойства монокристалла $\mathrm{EuBaCo}_{2-x} \mathrm{O}_{5.5-\delta}$. Ранее мы исследовали магнитные свойства монокристалла $\mathrm{GdBaCo}_{1.86} \mathrm{O}_{5.32}$, в котором также присутствуют только ионы $\mathrm{Co}^{3+}[10]$. Проведено сравнение магнитных свойств этих монокристалла и поликристаллов соответствующего состава в области $T=200-650 \mathrm{~K}$.

\section{2. Образцы и методики эксперимента}

Поликристаллические образцы $\mathrm{EuBaCo}_{2-x} \mathrm{O}_{5.5-\delta}[11]$ были синтезированы методом Печини [12]. Монокристалл $\mathrm{EuBaCo}{ }_{1.9} \mathrm{O}_{5.36}$ был выращен методом бестигельной зонной плавки на установке УРН-2-3П [10]. Элементный анализ образцов выполнен на сканирующем электронном микроскопе Inspect F (FEI) с энергодисперсионным спектрометром EDAX. Абсолютное содержание кислорода в исследуемых образцах было определено методом прямого восстановления водородом до исходных оксидов $\mathrm{Eu}_{2} \mathrm{O}_{3}, \mathrm{BaO}$ и металлического кобальта. Расчеты кристаллической структуры и уточнение структурных параметров по результатам рентгенографических исследований, проведенных на рентгеновском дифрактометре ДРОН-2 $\left(\mathrm{Cr} K_{\alpha}\right)$, осуществлялись с использованием программного пакета PowderCell 2.3 [13]. Установлено, что направление роста монокристалла совпадает с осью [120] в орторомбическом представлении Ртmm, направление [001] перпендикулярно оси роста. Образец легко колется вдоль плоскости (001). Состав образцов, параметры и объем элементарной ячейки представлены в табл. 1. Магнитные измерения проводились на магнитных весах с чувствительностью $\chi \approx 10^{-8} \mathrm{emu} / \mathrm{g}$ в температурной области $T=200-650 \mathrm{~K}$. Масса образцов для магнитных измерений составляла $14-30 \mathrm{mg}$, что уменьшало вероятность неоднородности состава. Электросопротивление образцов было измерено четырехконтактным методом с помощью автоматического контроллера Zirconia-M.

\section{3. Магнитоупорядоченная область}

Соединение $\mathrm{EuBaCo}_{2} \mathrm{O}_{5.50}$ имеет ряд магнитных переходов. Выше $200 \mathrm{~K}$ наблюдается переход из AF- в FM-состояние. На это указывают резкое увеличение намагниченности с максимумом вблизи $T \sim 250-260 \mathrm{~K}$ и наличие петель гистерезиса намагниченности ниже $T_{C}$.

Таблица 1. Состав, параметры и объем элементарной ячейки образцов $\mathrm{EuBaCo}_{2-x} \mathrm{O}_{5.50-\delta}$

\begin{tabular}{l|c|c|c|c}
\hline \multirow{2}{*}{ Состав } & \multicolumn{4}{|c}{ Структурные параметры } \\
\cline { 2 - 5 } & $a, \AA$ & $b, \AA$ & $c, \AA$ & $V, \AA^{3}$ \\
\hline $\mathrm{EuBaCo}_{2} \mathrm{O}_{5.50}$ & $3.880(7)$ & $7.827(3)$ & $7.546(7)$ & 229.23 \\
$\mathrm{EuBaCo}_{1.90} \mathrm{O}_{5.36}$ & $3.885(1)$ & $7.834(9)$ & $7.551(0)$ & 229.84 \\
$\mathrm{EuBaCo}_{1.90} \mathrm{O}_{5.36}$ & $3.884(1)$ & $7.833(2)$ & $7.552(4)$ & 229.74 \\
монокристалл & & & &
\end{tabular}
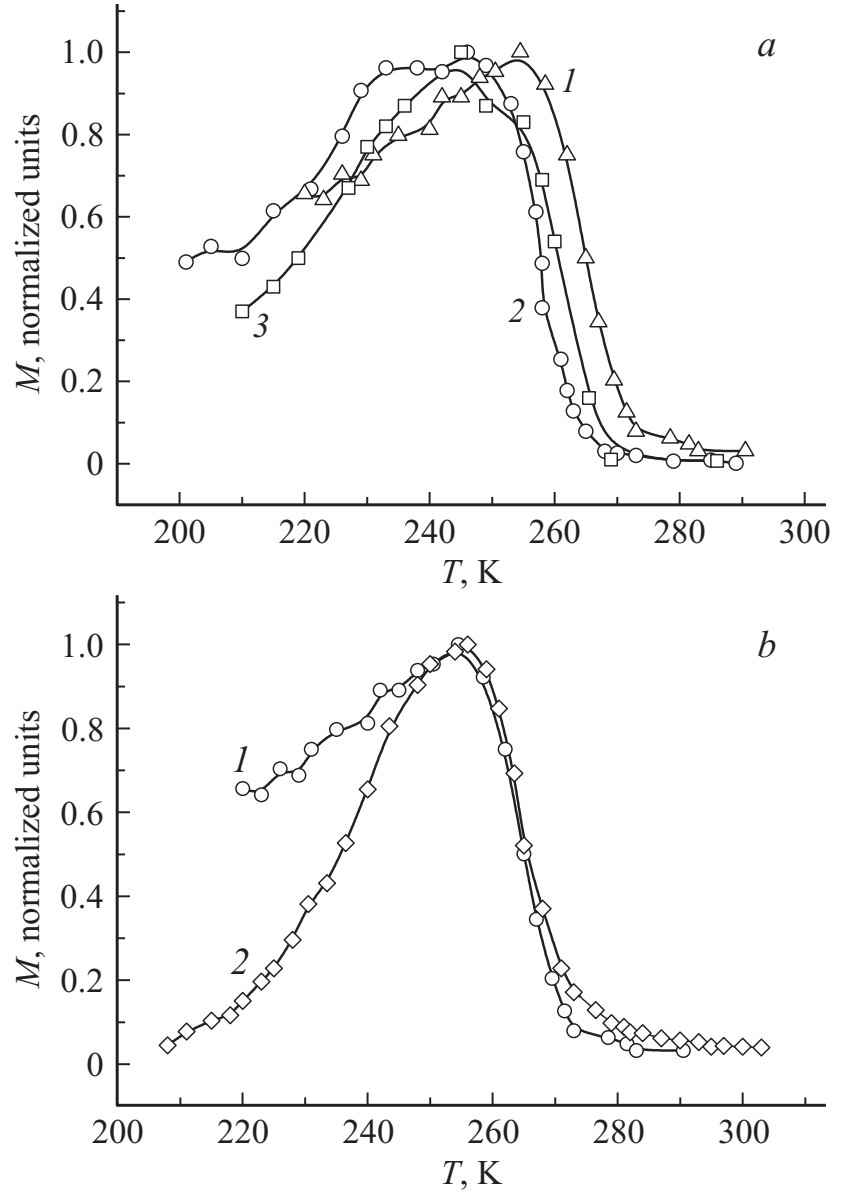

Рис. 1. Температурные зависимости приведенной намагниченности для $a-$ монокристалла и поликристаллов в поле $H=90$ Oе: 1 - монокристалл $\mathrm{EuBaCo}_{1.90} \mathrm{O}_{5.36}, 2$ - поликристалл $\mathrm{EuBaCo}_{1.90} \mathrm{O}_{5.36}, 3$ - поликристалл $\mathrm{EuBaCo}_{2} \mathrm{O}_{5.50}$. $b$ - монокристалла $\mathrm{EuBaCo}_{1.90} \mathrm{O}_{5.36}$ в полях $H: 1-90 \mathrm{Oе}$, и $2-450$ Oe.

При повышении температуры $T>300 \mathrm{~K}$ магнитный порядок нарушается и соединение переходит в парамагнитное состояние. Следует отметить, что наблюдаемая нелинейная температурная зависимость обратной парамагнитной восприимчивости $1 / \chi(T)$ с выпуклостью от оси $T$ и отрицательное значение асимптотической температуры Кюри $T_{a}$ обычно характерны для ферримагнетиков, в которых присутствует конкуренция между FM- и АF-взаимодействиями [14]. Ферримагнитное упорядочение спинов в $L n \mathrm{BaCo}_{2} \mathrm{O}_{5.50}$ не противоречит симметрийному структурному анализу [15] и экспериментальным данным по магнитным свойствам [16].

Для выяснения влияния структурных искажений и числа связей $\mathrm{Co}^{3+}-\mathrm{O}-\mathrm{Co}^{3+}$ на ферромагнитный вклад в намагниченность при сохранении средней степени окисления ионов кобальта $\mathrm{Co}^{3+}$, проведены измерения магнитной восприимчивости $\chi=M / H$ в слабых магнитных полях. На рис. 1, $a$ представлены температурные зависимости приведенной намагниченности для поликристаллов $\mathrm{EuBaCo}_{2} \mathrm{O}_{5.50}, \mathrm{EuBaCo}_{1.90} \mathrm{O}_{5.36}$ и монокри- 


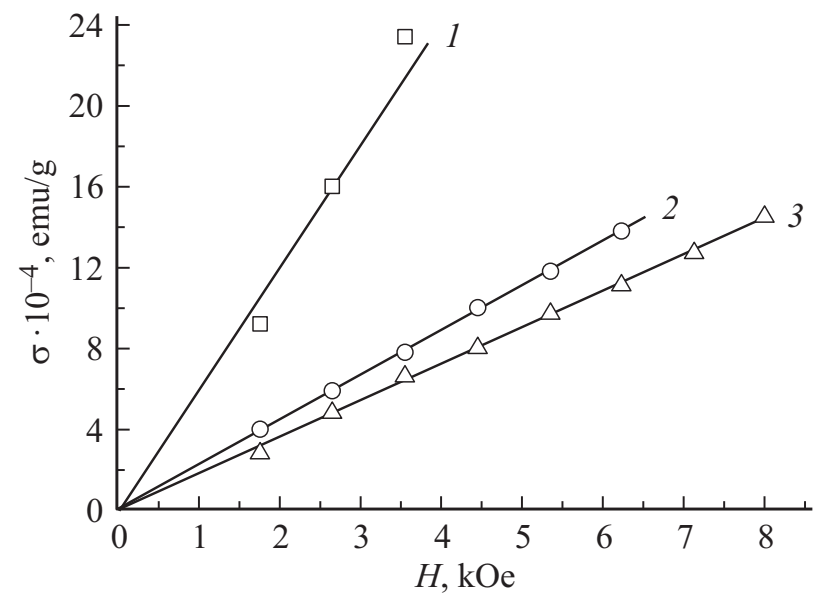

Рис. 2. Полевые зависимости намагниченности для монокристалла $\mathrm{EuBaCo}_{1.90} \mathrm{O}_{5.36}$ при разных температурах: $1-294 \mathrm{~K}$, $2-370 \mathrm{~K}, 3-500 \mathrm{~K}$.

сталла $\mathrm{EuBaCo}_{1.90} \mathrm{O}_{5.36}$ в поле $H=90$ Ое. Учитывая, что для монокристаллов $L n \mathrm{BaCo}_{2} \mathrm{O}_{5.50}$ наблюдается сильная анизотропия намагниченности вдоль осей $a, b$ и $c$ [1], в наших измерениях монокристалла магнитное поле было направлено вдоль направления [120]. Температура Кюри $T_{C}$ оценивалась по максимуму намагниченности, как общепринято для слоистых кобальтитов. Для поликристаллов стехиометрического состава $\mathrm{EuBaCo}_{2} \mathrm{O}_{5.50}$ и со структурными искажениями $\mathrm{EuBaCo}_{1.90} \mathrm{O}_{5.36}$ положения максимумов $M(T)$ совпадают, что указывает на нарушение FM-порядка при одной и той же темпеpaтyре $T=246 \mathrm{~K}$ независимо от числа $\mathrm{Co}^{3+}-\mathrm{O}-\mathrm{Co}^{3+}$ связей. В монокристалле максимум намагниченности расположен при более высокой температуре $T_{C}=255 \mathrm{~K}$. Эти значения не сильно отличаются и согласуются с литературными данными $T_{C}=255-265 \mathrm{~K}$ для $\mathrm{EuBaCo}_{2} \mathrm{O}_{5.50}[17,18]$.

На рис. $1, b$ представлены температурные зависимости намагниченности в полях $H=90$ и 450 Ое для монокристалла $\mathrm{EuBaCo}_{1.90} \mathrm{O}_{5.36}$. Видно, что внешнее магнитное поле по-разному влияет на величину намагниченности ниже $T_{C}$. При увеличении поля намагниченность сохраняется до более низких температур аналогично легированным кобальтитам $L n_{1-x} \mathrm{Ca}_{x} \mathrm{BaCo}_{2} \mathrm{O}_{5.50}[19,20]$. Такое поведение $M(H)$ ниже $T_{C}$ в слоистых кобальтитах со структурными искажениями часто объясняют состоянием кластерного стекла $[16,21]$. Резкое падение намагниченности выше $T_{C}$ связано с ослаблением FM-корреляций, когда $\sum n I S_{1} S_{2} \leq k T$, где $n-$ число обменно связанных пар, $I-$ обменный параметр, $S_{1}$ и $S_{2}-$ спины взаимодействующих ионов. Полевые зависимости намагниченности монокристалла выше $290 \mathrm{~K}$ являются линейными функциями приложенного поля, что характерно для парамагнитного или антиферромагнитного состояний (рис. 2). В работе [2] полагают, что в области $T=150-250 \mathrm{~K}$ в $\operatorname{EuBaCo}_{2} \mathrm{O}_{5.50 \pm \delta}(\delta=0.02)$ реализуется скошенный $\mathrm{AF}$, когда спины в антиферромагнитных подрешетках направлены под определенным углом к оси антиферромагнетизма. На наш взгляд, в исследованных кобальтитах в области $T>150 \mathrm{~K}$ устанавливается неколлинеарность магнитных моментов в антиферромагнитных подрешетках $\mathrm{Co}^{3+} \mathrm{IS}-\mathrm{O}-\mathrm{IS}$ или $\mathrm{HS}-\mathrm{O}-\mathrm{HS}$, а именно, реализуется треугольная конфигурация магнитных моментов Яфета-Киттеля [14]. Величина углов обменных связей под действием приложенного поля может изменяться. При повышении температуры $T>250 \mathrm{~K}$ происходит переход от треугольной конфигурации магнитных моментов ионов $\mathrm{Co}^{3+}$ к коллинеарному AF-упорядочению, поскольку непосредственный переход из неколлинеарного упорядочения спинов в парамагнитное состояние невозможен.

\section{4. Парамагнитная область}

Слоистые кобальтиты $\operatorname{LnBaCo}{ }_{2-x} \mathrm{O}_{5.50 \pm \delta}$ выше $300 \mathrm{~K}$ переходят в парамагнитное состояние. По виду температурной зависимости обратной восприимчивости $1 / \chi(T)$ можно судить о типе обменных взаимодействий (FM или $\mathrm{AF})$ в магнитоупорядоченной области и о спиновом состоянии магнитных ионов в парамагнитной области. На рис. 3 представлены температурные зависимости $1 / \chi(T)$ в области $T=300-650 \mathrm{~K}$ для исследованных образцов.

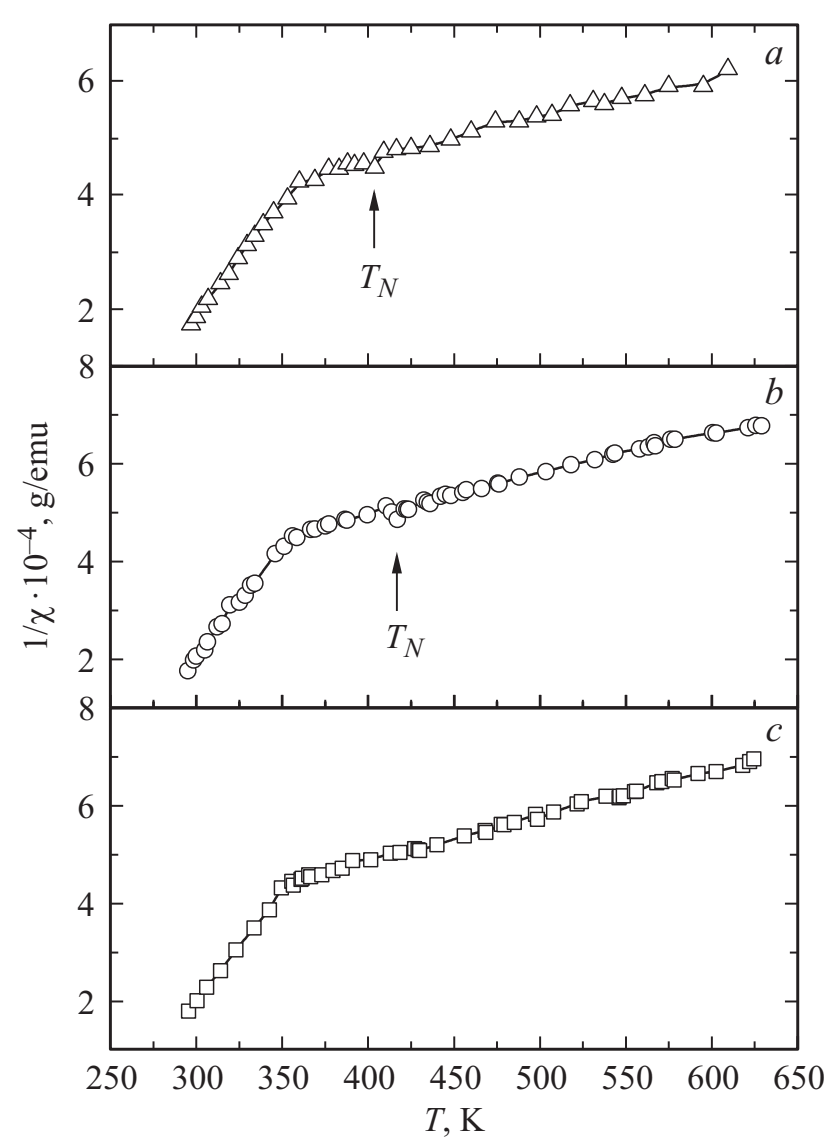

Рис. 3. Температурные зависимости обратной восприимчивости в поле $H=2.65 \mathrm{kOe}$ для образцов $\mathrm{EuBaCo}_{2-x} \mathrm{O}_{5.5-\delta}$ : $a-$ монокристалл $\mathrm{EuBaCo}_{1.90} \mathrm{O}_{5.36}, \quad b-$ поликристалл $\mathrm{EuBaCo}_{1.90} \mathrm{O}_{5.36}, c-$ поликристалл $\mathrm{EuBaCo}_{2} \mathrm{O}_{5.50}$. 
Таблица 2. Экспериментальные и расчетные значения эффективного магнитного момента $\mu_{\mathrm{eff}}$ для образцов $\mathrm{EuBaCo}_{2-x} \mathrm{O}_{5.5-\delta}$ при $T>470 \mathrm{~K}$

\begin{tabular}{|c|c|c|c|}
\hline Состав & $\mathrm{EuBaCo}_{2} \mathrm{O}_{5.50}$ & $\mathrm{EuBaCo}_{1.90} \mathrm{O}_{5.36}$ & $\begin{array}{c}\mathrm{EuBaCo}_{1.90} \mathrm{O}_{5.36} \\
\text { монокристалл }\end{array}$ \\
\hline Эксперимент: $\mu_{\mathrm{eff}}, \mu_{\mathrm{B}}$ & 6.53 & 6.49 & 7.38 \\
\hline Распределение ионов $\mathrm{Co}^{3+}$ & \multicolumn{3}{|c|}{ Расчетные значения $\mu_{\mathrm{eff}}, \mu_{\mathrm{B}}$} \\
\hline $\begin{array}{l}(\mathrm{IS})_{\mathrm{pyr}}+[0.5(\mathrm{LS})+0.5(\mathrm{HS})]_{\mathrm{oct}} \\
(\mathrm{IS})_{\mathrm{pyr}}+(\mathrm{IS})_{\mathrm{oct}} \\
(\mathrm{IS})_{\mathrm{pyr}}+[0.5(\mathrm{IS})+0.5(\mathrm{HS})]_{\mathrm{oct}} \\
(\mathrm{IS})_{\mathrm{pyr}}+(\mathrm{HS})_{\mathrm{oct}} \\
(\mathrm{HS})_{\mathrm{pyr}}+[0.5(\mathrm{LS})+0.5(\mathrm{HS})]_{\mathrm{oct}} \\
(\mathrm{HS})_{\mathrm{pyr}}+[0.5(\mathrm{IS})+0.5(\mathrm{HS})]_{\mathrm{oct}} \\
(\mathrm{HS})_{\mathrm{pyr}}+(\mathrm{HS})_{\mathrm{oct}}\end{array}$ & $\begin{array}{l}5.97 \\
5.66 \\
6.23 \\
6.85 \\
7.21 \\
7.40 \\
7.87\end{array}$ & & \\
\hline
\end{tabular}

В большинстве работ магнитные свойства кобальтитов изучались в области $T \leq 400 \mathrm{~K}$. Нам известно несколько работ, в которых приводятся данные по восприимчивости при высоких температурах $[19,20,22,23]$. Из рис. 3 видно, что зависимости $1 / \chi(T)$ имеют характерный для ферримагнетиков гиперболический вид с выпуклостью от оси $T$, в которых конкурируют FM- и AF-взаимодействия [14]. На присутствие FM-взаимодействий указывают резкое увеличение намагниченности и петли гистерезиса в области $T \leq 260 \mathrm{~K}[2,18]$, а на AF-обмен - отрицательное значение асимптотической температуры $T_{a}$, полученной экстраполяцией $1 / \chi(T)$ из далекой парамагнитной области. В области $T \approx 360 \mathrm{~K}$ наблюдается резкое изменение наклона зависимости $1 / \chi(T)$, которое ряд авторов связывает с переходом изолятор-металл из-за орбитального упорядочения IS-состояний ионов $\mathrm{Co}^{3+}[5]$. При этой температуре магнитный момент ионов $\mathrm{Co}^{3+}$ $(S=1)$ не изменяется, а вклад свободных носителей заряда очень мал. Изменение наклона обратной восприимчивости вблизи $360 \mathrm{~K}$ связано с конкуренцией FMи AF-обменных взаимодействий в ферримагнетиках в области ближнего магнитного порядка.

Для стехиометрического поликристалла $\mathrm{EuBaCo}_{2} \mathrm{O}_{5.50}$ температурная зависимость парамагнитной восприимчивости $1 / \chi(T)$ имеет типичный вид для коллинеарных ферримагнетиков, в которых FM- и AF-магнитные упорядочения нарушаются при одной и той же температуре $\left(T_{C}=T_{N}\right)$. В слоистых кобальтитах $\mathrm{EuBaCo}_{1.90} \mathrm{O}_{5.36} \mathrm{c}$ вакансиями в кобальтовой и кислородной подрешетках вблизи $400 \mathrm{~K}$ наблюдается максимум восприимчивости (рис. 4). Такое поведение $\chi(T)$ указывает на сохранение антиферромагнитного порядка выше $T_{C}$ в ферримагнетиках, в которых $T_{N}>T_{C}$ [14]. В работе [24] было показано, что в $\mathrm{EuBaCo}_{2-x} \mathrm{O}_{5.5-\delta}$ вблизи одной элементарной ячейки с вакансией кобальта формируется кластер из 7-8 ближайших ячеек со структурными искажениями. По-видимому, структурные искажения способствуют сохранению антиферромагнитного порядка до более высоких температур при наложении магнитного поля. Аналогичный максимум восприимчивости выше

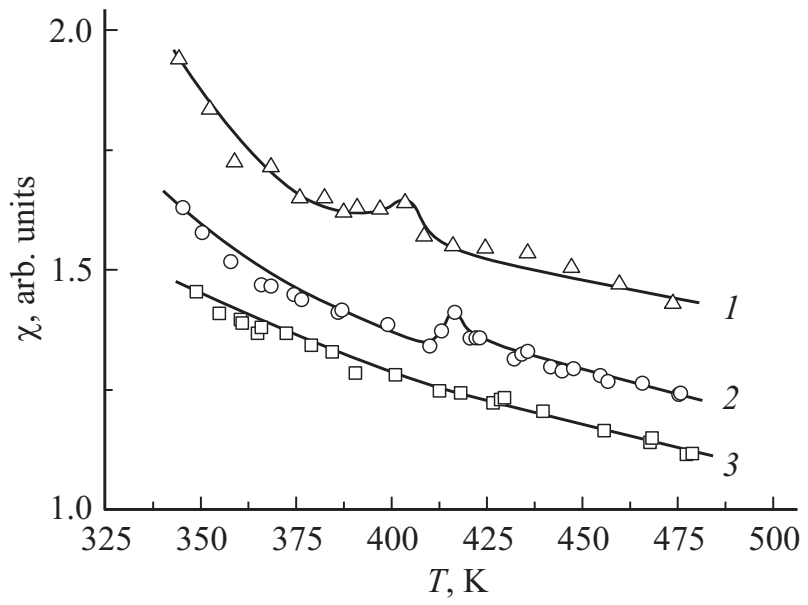

Рис. 4. Температурные зависимости магнитной восприимчивости в магнитном поле $H=2.65 \mathrm{kOe}$ для образцов $\mathrm{EuBaCo}_{2-x} \mathrm{O}_{5.5-\delta}: 1$ - монокристалл $\mathrm{EuBaCo}_{1.90} \mathrm{O}_{5.36}, 2$ - поликристалл $\mathrm{EuBaCo}_{1.90} \mathrm{O}_{5.36}, 3$ - поликристалл $\mathrm{EuBaCo}_{2} \mathrm{O}_{5.50}$.

$T_{C}$ наблюдался в монокристалле $\mathrm{GdBaCo}_{1.86} \mathrm{O}_{5.32}$ [10] и в легированном $\mathrm{Y}\left(\mathrm{Ba}_{0.9} \mathrm{Ca}_{0.1}\right) \mathrm{Co}_{2} \mathrm{O}_{5.50}$ [23], в которых также присутствуют структурные искажения.

В парамагнитной области, когда магнитные ионы не взаимодействуют друг с другом, восприимчивость описывается законом Кюри-Вейсса

$$
\chi=\frac{C}{T-\theta}=\frac{N \mu_{\mathrm{eff}}^{2} \mu_{\mathrm{B}}^{2}}{3 k(T-\theta)}
$$

где $C$ - постоянная Кюри-Вейсса, $\theta-$ асимптотическая температура Кюри, $N$ - число Авагадро, $\mu_{\mathrm{eff}}-$ эффективный магнитный момент, $\mu_{\mathrm{B}}-$ магнетон Бора, $k$ - постоянная Больцмана. В слоистых кобальтитах эффективный магнитный момент определяется суммой вкладов магнитных ионов

$$
\mu_{\text {eff }}^{2}=\mu_{\text {eff }}^{2}(\mathrm{Eu})+2\left[n_{1} \mu_{\text {eff }}^{2}+n_{2} \mu_{\mathrm{eff}}^{2}\right],
$$

где $n_{1}$ и $n_{2}-$ концентрация ионов $\mathrm{Co}^{3+}(\mathrm{IS})$ и $\mathrm{Co}^{3+}(\mathrm{HS})$. Ионы $\mathrm{Eu}^{3+}$ при $T=0 \mathrm{~K}$ являются немагнитными. Од- 


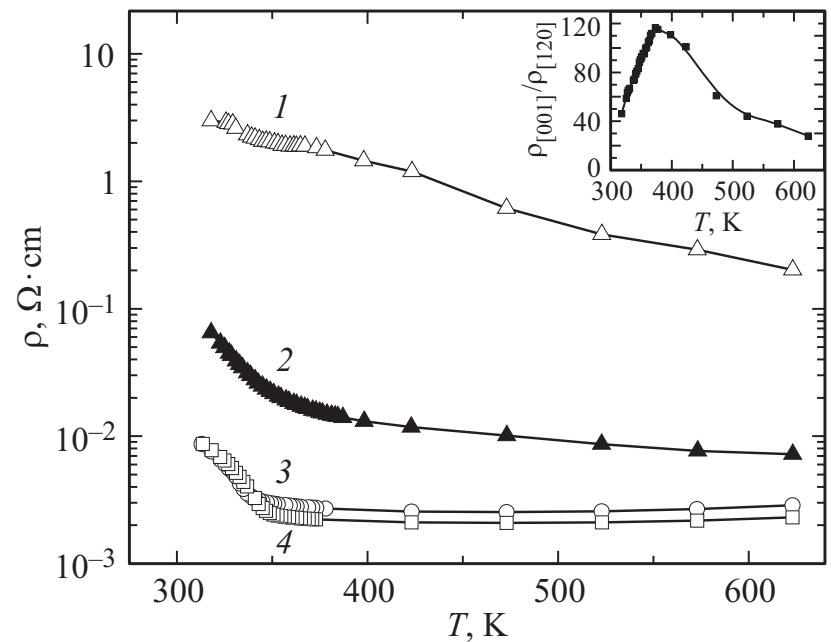

Рис. 5. Температурные зависимости электросопротивления для образцов $\mathrm{EuBaCo}_{2-x} \mathrm{O}_{5.5-\delta}: 1-$ монокристалл $\mathrm{EuBaCo}_{1.90} \mathrm{O}_{5.36}$, электросопротивление вдоль направления [001], 2 - монокристалл $\mathrm{EuBaCo}_{1.90} \mathrm{O}_{5.36}$, электросопротивление вдоль направления [120], 3 - поликристалл $\mathrm{EuBaCo}_{1.90} \mathrm{O}_{5.36}, 4$ - поликристалл $\mathrm{EuBaCo}_{2} \mathrm{O}_{5.50}$. На вставке приведено отношение электросопротивления вдоль направлений $\rho[001] / \rho[120]$ для монокристалла $\mathrm{EuBaCo}_{1.90} \mathrm{O}_{5.36}$.

нако в области $T=400-625 \mathrm{~K}$ за счет Ван-Флековских возбуждений эффективный магнитный момент $\mathrm{Eu}^{3+}$ возрастает от $\mu_{\text {eff }}=3.7 \mu_{\mathrm{B}}$ до $\mu_{\mathrm{eff}}=4.0 \mu_{\mathrm{B}}$ [25]. Это необходимо учитывать при расчете экспериментальных

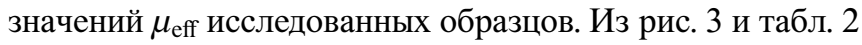
видно, что в области $T=480-630 \mathrm{~K}$ в поликристаллах зависимости $1 / \chi(T)$ почти совпадают. Поскольку в $\mathrm{EuBaCo}_{1.90} \mathrm{O}_{5.36}$ имеются Со вакансии по сравнению со стехиометрическим $\mathrm{EuBaCo}_{2} \mathrm{O}_{5.50}$, близкие значения $\chi(T)$ для этих образцов могут быть обусловлены вакансиями в октоузлах, занятыми ионами $\mathrm{Co}^{3+}$ в LS-состоянии. Линейные зависимости $1 / \chi(T)$ в этой области температур указывают на сохранение спиновых состояний ионов $\mathrm{Co}^{3+}$

Монокристалл $\mathrm{EuBaCo}_{1.90} \mathrm{O}_{5.36}$ в парамагнитной области имеет бо́льшие значения $\chi(T)$ и $\mu_{\text {eff }}$ по сравнению с поликристаллом соответствующего состава. Число структурных дефектов в них одинаково, поэтому они не должны влиять на величину $\chi(T)$. Существенным отличием в измерениях восприимчивости является то, что в монокристалле магнитное поле было направлено вдоль направления [120]. Учитывая сильную анизотропию намагниченности вдоль направлений $a, b$ и $c$ в магнитоупорядоченной области [1], можно предположить, что магнитные моменты ионов $\mathrm{Co}^{3+}$ расположены в плоскости (001), т.е. реализуется трехмерная $\mathrm{XY-модель} \mathrm{[26],} \mathrm{в} \mathrm{которой} \mathrm{взаимодействия} \mathrm{между} \mathrm{иона-}$ ми $\mathrm{Co}^{3+}$ вдоль оси $c$ значительно слабее по сравнению со взаимодействиями в перпендикулярном направлении. Подтверждением XY-модели может служить сильная анизотропия $g$-фактора, однако экспериментальные данные для $g$-фактора ионов $\mathrm{Co}^{3+}$ в литературе отсутству- ют. В парамагнитной области приложенное магнитное поле действует независимо на каждый магнитный момент. Отметим, что в монокристалле наклон зависимости $1 / \chi(T)$ меньше и соответственно эффективный магнитный момент больше. Большое значение $\mu_{\mathrm{eff}}$ в монокристалле может быть связано с изменением полной энергии при наложении магнитного поля и изменением спиновых состояний. Магнитное поле, приложенное вдоль плоскости $a b$, может способствовать спиновым переходам ионов $\mathrm{Co}^{3+} \mathrm{LS} \rightarrow \mathrm{IS}$ и IS $\rightarrow \mathrm{HS}$. Подтверждением анизотропии магнитных взаимодействий могут служить данные по электросопротивлению. На рис. 5 представлены температурные зависимости электросопротивления для поликристаллов и монокристалла вдоль направлений [001] и [120]. Видно, что в исследованной области температур перенос носителей заряда по $e_{g}$ уровню осуществляется легче в плоскости $a b$. На отношении электросопротивления вдоль направлений $\rho[001] / \rho[120]$ для монокристалла $\mathrm{EuBaCo}_{1.90} \mathrm{O}_{5.36}$ (см. вставку к рис. 5) при температуре $T \sim 375 \mathrm{~K}$ проявляется максимум.

\section{5. Спиновые состояния ионов $\mathrm{Co}^{3+}$ В парамагнитной области}

Из линейных температурных зависимостей обратной восприимчивости в далекой парамагнитной области по закону Кюри-Вейсса можно определить экспериментальные значения эффективного магнитного момента исследуемых соединений (1). В области температур $\left(T_{1}-T_{2}\right)$ постоянная Кюри и эффективный магнитный момент описываются выражением

$$
C=\frac{\left(T_{1}-T_{2}\right) M}{\chi_{1}^{-1}-\chi_{2}^{-1}},
$$

где $M$ - молярная масса. Изменение наклона зависимости $1 / \chi(T)$ указывает на изменение спинового состояния магнитных ионов. При расчете теоретических значений $\mu_{\text {eff }}$ для разных спиновых состояний ионов $\mathrm{Co}^{3+}$ в $\mathrm{EuBaCo}_{2-x} \mathrm{O}_{5.5-\delta}$ используется формула 2. В табл. 2 приведены расчетные и экспериментальные значения эффективного магнитного момента для исследованных образцов при $T>470 \mathrm{~K}$. Из таблицы видно, что экспериментальные значения $\mu_{\text {eff }}$ для поликристаллов близки. Несколько меньшее значение $\mu_{\text {eff }}$ в $\mathrm{EuBaCo}_{1.90} \mathrm{O}_{5.36}$ по сравнению с $\mathrm{EuBaCo}_{2} \mathrm{O}_{5.50}$ может быть связано с вакансиями в кобальтовой подрешетке. Общепринятым считается следующее распределение ионов Со при низких температурах: в пирамидах - IS состояния, а в октоузлах $0.5(\mathrm{LS})+0.5(\mathrm{HS})$ [19,22,27]. Данные по ЯMP в $\mathrm{EuBaCo}_{2} \mathrm{O}_{5.50}$ подтверждают присутствие всех трех спиновых состояний LS, IS, HS ионов $\mathrm{Co}^{3+}$ при комнатной температуре [8]. При повышении температуры и при увеличении объема элементарной ячейки могут происходить переходы части ионов $\mathrm{Co}^{3+}$ в более высокоспиновое состояние из-за сближения их полной энергии [5]. В стехиометрическом поликристалле 
$\mathrm{EuBaCo}_{2} \mathrm{O}_{5.50}$ значение $\mu_{\text {eff }}$ соответствует ситуации, когда 63\% ионов кобальта находятся в IS-состоянии, а $37 \%$ имеют высокоспиновое состояние. В $\mathrm{EuBaCo}_{1.90} \mathrm{O}_{5.36}$ присутствуют $60 \%$ IS + 40\% HS-состояний ионов $\mathrm{Co}^{3+}$ от номинальной концентрации кобальта. Следовательно, несмотря на вакансии в кобальтовых подрешетках в $\mathrm{EuBaCo}_{1.90} \mathrm{O}_{5.36}$, соотношение числа IS- и HS-состояний довольно близкое и вакансии равномерно распределены в местах $\mathrm{Co}^{3+}(\mathrm{LS})$.

В монокристалле $\mathrm{EuBaCo}_{1.90} \mathrm{O}_{5.36}$ экспериментальное значение $\mu_{\mathrm{eff}}=7.38 \mu_{\mathrm{B}}$ существенно выше $\mu_{\mathrm{eff}}=6.49 \mu_{\mathrm{B}}$ для поликристалла такого же состава. Наблюдаемая в монокристалле величина $\mu_{\text {eff }}$ соответствует содержанию $\approx 20 \%$ ионов $\mathrm{Co}^{3+}$ (IS) и $\approx 80 \%$ ионов $\mathrm{Co}^{3+}$ (HS). Это соотношение спиновых состояний указывает, что переход в HS-состояние происходит не только в октоузлах, но и в пирамидах. В парамагнитной области $T>470 \mathrm{~K}$ в пирамидальном окружении находятся 30\% HS-состояний и $20 \%$ IS-состояний от общего числа $\mathrm{Co}^{3+}$ ионов. В работе [24] показано, что дефицит кобальтовых ионов и связанного с ним кислорода приводит к увеличению числа пирамидального окружения. Это может являться одной из причин появления HS-состояний в пирамидах. В парамагнитной области магнитное поле действует на каждый магнитный момент независимо. В монокристалле внешнее магнитное поле было направлено вдоль плоскости $a b$. Заметное различие значений $\mu_{\mathrm{eff}}$ для монокристалла и поликристалла одинакового состава может указывать на направление спинов ионов $\mathrm{Co}^{3+}$ в этой плоскости. Такое предположение согласуется с сильной анизотропией намагниченности в области температур $T=200-300 \mathrm{~K}$ вдоль осей $a, b, c$ [1] и с выводами в работе [15]. Отметим, что в монокристалле переход ионов Со в состояние Хунда $(S=2)$ осуществляется при более низких температурах по сравнению с поликристаллом.

\section{6. Обсуждение результатов}

Экспериментальные данные по магнитным свойствам кобальтитов $\mathrm{EuBaCo}_{2-x} \mathrm{O}_{5.5-\delta}$ показали, что они являются ферримагнетиками, в которых конкурируют FM- и AF-обменные взаимодействия. Вакансии в кобальтовых и кислородных подрешетках при сохранении валентности $\mathrm{Co}^{3+}$ приводят к сохранению AF-упорядочения до более высоких температур по сравнению с FM-порядком $\left(T_{N}>T_{C}\right)$. В литературе преобладает точка зрения, что в системе $L n \mathrm{BaCO}_{2-x} \mathrm{O}_{5.50 \pm \delta}$ в температурной области $200<T<300 \mathrm{~K}$ присутствуют две коллинеарные магнитные фазы, а именно, ферромагнитные области (FM-кластеры), внедренные в антиферромагнитную матрицу. В этом случае в парамагнитной области температурная зависимость обратной восприимчивости должна иметь линейный вид или быть вогнута к оси температур, что не согласуется с нашими экспериментальными данными (рис. 3). На наш взгляд, слоистые кобальтиты являются ферримагнетиками, в которых имеется несколько (более 2) магнитных подрешеток с FM- и AF-взаимодействиями. Не исключена вероятность неколлинеарного упорядочения этих подрешеток. Сложность магнитной системы $\mathrm{EuBaCo}_{1.90} \mathrm{O}_{5.36}$ обусловлена слоистостью кристаллической структуры, в которой слои $\mathrm{Co}-\mathrm{O}$ разделены немагнитными $\mathrm{Ba}-\mathrm{O}$ и $\mathrm{Eu}-\mathrm{O}$ слоями, разной симметрией окружения ионов $\mathrm{Co}^{3+}$ и изменением их магнитного момента при повышении температуры и уменьшении параметров решетки.

Поликристаллы $\mathrm{EuBaCo}_{2} \mathrm{O}_{5.50}$ и $\mathrm{EuBaCo}_{1.90} \mathrm{O}_{5.36}$ имеют одинаковое значение $T_{C}$. Ферромагнитная температура Кюри зависит от величины обменного параметра $J$ и числа взаимодействующих IS-HS пар. Обменная энергия определяется выражением

$$
E_{e x}=2 J S_{1} S_{2} \cos \theta
$$

т.е. зависит от расстояния и угла $\theta$ для обменных связей $\mathrm{Co}^{3+}(\mathrm{IS})-\mathrm{O}-\mathrm{Co}^{3+}(\mathrm{HS})$. Обменный параметр не зависит от температуры. Близкие области температур, где наблюдаются максимальные значения намагниченности в этих образцах (рис. 1), указывают на одинаковое число FM обменно-связанных пар. Расстояния $\mathrm{Co}-\mathrm{O}$ связей в пирамидах и октаэдрах близки [22,24], поэтому FM-взаимодействия $\mathrm{Co}-\mathrm{O}-\mathrm{Co}$ могут осуществляться между ионами Сo, расположенными в пирамидах и в октаэдрах. Как отмечалось выше, при комнатной температуре могут присутствовать все три спиновых состояния. Вакансии в Со подрешетке в $\mathrm{EuBaCo}_{1.90} \mathrm{O}_{5.36}$ не влияют на значение $T_{C}$, что может указывать на преимущественное расположение вакансий в октопозициях. В области $T \leq 400 \mathrm{~K}$ изменяется соотношение числа FM и AF обменных связей. Этим объясняется большое отрицательное значение асимптотической температуры Кюри $\theta$ и появление максимума восприимчивости в поликристалле и монокристалле $\mathrm{EuBaCo}_{1.90} \mathrm{O}_{5.36}$ выше $T_{C}$ (рис. 4). Небольшая величина максимума $\chi(T)$ связана с тем, что восприимчивость антиферромагнитной фазы $\chi \approx 10^{-6} \mathrm{emu} / \mathrm{g}$ существенно меньше значений $\chi \approx 10^{-2}-10^{-4} \mathrm{emu} / \mathrm{g}$ для ферромагнетиков. Вакансии в кобальтовой подрешетке и связанные с ними вакансии кислорода приводят к увеличению числа пирамидальных позиций, и соответственно к увеличению числа ближайших AF пар ионов $\mathrm{Co}^{3+}-(\mathrm{IS}-\mathrm{IS})$ или (HS-HS) [4]. При повышении температуры происходят постепенные переходы спиновых состояний ионов $\mathrm{Co}^{3+}$. В парамагнитной области $T>T_{N}$ или $T_{C}$, когда обменная энергия меньше тепловой энергии $k T$, ионы $\mathrm{Co}^{3+}$ становятся независимыми друг от друга. Несмотря на разную концентрацию ионов кобальта в $\mathrm{EuBaCo}_{2} \mathrm{O}_{5.50}$ и $\mathrm{EuBaCo}_{1.90} \mathrm{O}_{5.36}$ поликристаллах, соотношение числа ионов $\mathrm{Co}^{3+}$ в ISи HS-состояниях одинаковое. В монокристалле и поликристалле $\mathrm{EuBaCo}_{1.90} \mathrm{O}_{5.36}$ значения эффективного магнитного момента $\mu_{\text {eff }}$ в далекой парамагнитной области $T>500 \mathrm{~K}$ сильно отличаются. Это может свидетельствовать о понижении температуры спиновых переходов IS-HS при направлении внешнего поля вдоль плоскости $a b$. Небольшие увеличение $T_{C}$ и уменьшение $T_{N}$ в 
монокристалле по сравнению с поликристаллом можно объяснить уменьшением вклада AF-взаимодействий между ближайшими соседями по сравнению с FM-вкладом. В коллинеарных антиферромагнетиках анизотропия составляющих восприимчивости $\left(\chi_{\|}\right.$и $\left.\chi_{\perp}\right)$ обычно наблюдается в магнитоупорядоченной области. Максимум параллельной и перпендикулярной составляющих намагниченности $M=\chi H$ проявляется при одной и той же температуре $T_{N}$ [14]. В слоистых соединениях с кристаллографической точки зрения взаимодействия в слоях существенно сильнее, чем между слоями. В них возможна ситуация, когда значения $\chi_{\perp}$ меньше $\chi_{\|}$, а положения максимума для $\chi_{\perp}(T)$ и $\chi_{\|}(T)$ могут не совпадать [26]. Менее выраженный максимум восприимчивости и его смещение в сторону низких температур в монокристалле $\mathrm{EuBaCo}_{1.90} \mathrm{O}_{5.36}$ по сравнению с поликристаллом того же состава могут быть обусловлены вкладом $\chi_{\perp}$ составляющей, когда спины $\mathrm{Co}^{3+}$ ионов лежат в плоскости $a b$.

Для трактовки экспериментальных данных по магнитным свойствам слоистых кобальтитов необходимы правильное определение размерности магнитной системы (3D, 2D или 1D) и выбор модели (Гейзенберг, Изинг или XY-модель). В низкоразмерных магнитных системах (1D и 2D) эффекты ближнего порядка являются определяющими. В бесконечно длинной цепочке (1D) эффект дальнего магнитного порядка может быть только при $T=0$. В 2D-системах при появлении межцепочечных взаимодействий возможен дальний магнитный порядок, однако во всех известных 2D соединениях он проявляется при низких температурах $T<20 \mathrm{~K}$ [26]. В слоистых кобальтитах $\mathrm{EuBaCo}_{2-x} \mathrm{O}_{5.5-\delta}$ дальний магнитный порядок наблюдается в области высоких температур $200<T<400 \mathrm{~K}$. Наиболее вероятной размерностью магнитной системы является $3 \mathrm{D}$ система благодаря слабому взаимодействию между Со-О слоями.

В теории молекулярного поля при рассмотрении обменных взаимодействий гамильтониан имеет вид

$$
H=-2 \sum\left[I_{x y}\left(S_{i x} S_{j x}+S_{i y} S_{j y}\right)+I_{z} S_{i z} S_{j z}\right] .
$$

Модель Гейзенберга применима для изотропного обменного взаимодействия, когда $I_{x y}=I_{z}$. В модели Изинга взаимодействия осуществляются вдоль одного направления, когда $I_{x y}=0$. В XY-модели спины лежат в $\mathrm{XY}$-плоскости $\left(I_{z}=0\right)$. Учитывая тот факт, что в слоистых кобальтитах вблизи $T_{C}$ намагниченность вдоль направлений $a$ и $b$ существенно больше намагниченности вдоль оси $c$, можно предположить, что в слоистых кобальтитах реализуется трехмерная ХY-модель. Следует отметить, что существует немного соединений, содержащих ионы $\mathrm{Co}^{3+}: \mathrm{LaCoO}_{3}$, в котором отсутствует дальний магнитный порядок, и $\mathrm{TlSr}_{2} \mathrm{CoO}_{5}[28,29]$. Перовскито-подобный слоистый $\mathrm{TlSr}_{2} \mathrm{CoO}_{5}$ показывает необычные магнитные свойства. В работе [29] установлено, что ниже $T_{N}=150 \mathrm{~K}$ реализуется антиферромагнитное упорядочение. В области $T=300-750 \mathrm{~K}$ выполняется закон Кюри-Вейсса с асимптотической температурой Кюри $\theta=200 \mathrm{~K}$, что указывает на ферромагнитный порядок ниже $200 \mathrm{~K}$ и парамагнитное состояние выше $300 \mathrm{~K}$ смеси $\sim 35 \%$ ионов $\mathrm{Co}^{3+}$ в HS-состоянии и $65 \%$ в IS-состоянии. Необычное поведение $\chi^{-1}(T)$ в области $T=150-250 \mathrm{~K}$ авторы объясняют структурными искажениями октаэдров и присутствием двух фаз: низкотемпературной парамагнитной $t$-фазы и высокотемпературной ферромагнитной $o$-фазы. Для шпинели $\mathrm{CoMn}_{2} \mathrm{O}_{4}$ предположительными формулами являются $\mathrm{Co}_{1-x}^{3+} \mathrm{Mn}_{x}^{2+}\left[\mathrm{Co}_{x}^{\mathrm{III}} \mathrm{Mn}_{2-x}^{3+}\right] \mathrm{O}_{4}$ или $\mathrm{Co}_{1-x}^{2+}+\mathrm{Mn}_{x}^{2+}\left[\mathrm{Co}_{x}^{\mathrm{III}} \mathrm{Mn}_{2-x}^{3+}\right] \mathrm{O}_{4}$, где $\mathrm{Co}^{3+}$ имеет $S=2$, а $\mathrm{Co}^{\mathrm{III}} S=1[4,14]$. Такое распределение ионов металла по октаэдрическим и тетраэдрическим узлам шпинели определено из данных по намагниченности, однако спиновые состояния Со ионов, полученные независимыми методами, неизвестны. Для моделей XY и Изинга характерна сильная анизотропия свойств. Хорошим индикатором анизотропии является $g$-фактор. Для соединений $\operatorname{Ln} \mathrm{BaCo}_{2} \mathrm{O}_{5.50 \pm \delta}$ такие данные отсутствуют. Известно малое число трехмерных XY-антиферромагнетиков. Эти соединения содержат ионы $\mathrm{Co}^{2+}$ в октаэдрическом окружении и имеют $g_{\perp} \gg g_{\|}[26]$. Поведение ионов $\mathrm{Co}^{2+}\left(d^{7}\right)$ чаще всего соответствует XY-модели или модели Изинга в зависимости от окружения иона, от знака и величины расщепления уровней в нулевом поле. Понижение симметрии координационной сферы нарушает эквивалентность $X$ - и $Y$-направлений. В результате может появиться скрытая неколлинеарность спинов.

\section{7. Выводы}

Полученные данные по магнитным свойствам слоистых кобальтитов $\mathrm{EuBaCo}_{2-x} \mathrm{O}_{5.5-\delta}$ показали, что в области $T=200-400 \mathrm{~K}$ присутствует не смесь двух коллинеарных AF- и FM-фаз, а реализуется трехмерное $\mathrm{XY-ферримагнитное} \mathrm{упорядочение} \mathrm{магнитных} \mathrm{подреше-}$ ток. На 3D-ферримагнетизм указывают вид зависимостей $1 / \chi(T)$ и сохранение антиферромагнитного порядка до более высоких температур $\left(T_{C}<T_{N}\right)$. ХY-модель обусловлена тем, что магнитный порядок в основном определяется взаимодействиями в плоскости $a b$, а обмен между этими плоскостями слабый из-за структурных особенностей кристаллической решетки слоистых кобальтитов. Следует отметить, что в области дальнего магнитного порядка для поликристалла $\mathrm{EuBaCo}_{1.90} \mathrm{O}_{5.36}$ значение $T_{C}=246 \mathrm{~K}$ немного ниже $T_{C}=254 \mathrm{~K}$ для монокристалла того же состава. Однако для температуры Нееля ситуация обратная, а именно в поликристалле $T_{N}=417 \mathrm{~K}$, а в монокристалле $T_{N}=406 \mathrm{~K}$. Такая инверсия значений $T_{C}$ и $T_{N}$ может быть связана с изменением числа AF и FM обменно-связанных ионов $\mathrm{Co}^{3+}$ при направлении внешнего магнитного поля вдоль плоскости $a b$. Вакансии в кобальтовой и кислородной подрешетках приводят к понижению симметрии окружения ионов Со в октаэдрическом окружении и увеличению объема элементарной ячейки по сравнению со стехиометрическим составом $\mathrm{EuBaCo}_{2} \mathrm{O}_{5.50}$, что влияет на спиновое состояние ионов $\mathrm{Co}^{3+}$ и на число АF- и FM-пар. В $\mathrm{EuBaCo}_{1.90} \mathrm{O}_{5.36}$ не исключена вероятность 
неколлинеарного упорядочения магнитных подрешеток, в которых спины лежат в плоскости $a b$. Такое предположение не противоречит наблюдаемым магнитным свойствам и симметрии кристаллической структуры.

\section{Список литературы}

[1] A.A. Taskin, A.N. Lavrov, Yoichi Ando. Phys. Rev. B 71, 134414 (2005).

[2] B. Raveau, M.D. Motin Seikh, V. Pralong, V. Caignaert. Bull. Mater. Sci. 32, 305 (2009).

[3] A. Maignan, C. Martin, D. Pelloquin, N. Nguyen, B. Raveau. J. Solid State Chem. 142, 247 (1999).

[4] Д.Б. Гуденаф. Магнетизм и химическая связь / Пер. с англ. / Под ред. Б.Е. Левина и С.С. Горелика. Металлургия, M. (1966). $328 \mathrm{c}$.

[5] M.A. Korotin, S.Yu. Ezhov, I.V. Solovyev, V.I. Anisimov, D.I. Khomskii, G.A. Sawatzky. Phys. Rev. B 54, 5309 (1996).

[6] D.D. Khalyavin. Phys. Rev. B 72, 134408 (2005).

[7] Yu.P. Chernenkov, V.P. Plakhty, V.I. Fedorov, S.N. Barilo, S.V. Shiryaev, G.L. Bychkov. Phys. Rev. B 71, 184105 (2005).

[8] H. Kubo, K. Zenmyo, M. Itoh, N. Nakayama, T. Mizota, Y. Ueda. JMMM 272-276, 581 (2004).

[9] M. Soda, Y. Yasui, T. Fujita, M. Sato, K. Kakurai. J. Phys.Soc. Jpn. 72, 1729 (2003).

[10] T.I. Arbuzova, S.V. Telegin, S.V. Naumov, E.I. Patrakov, O.G. Reznitskih. Solid State Phenom. 215, 83 (2014).

[11] Т.И. Арбузова, С.В. Наумов, С.В. Телегин. ФТТ 59, 517 (2017).

[12] M.P. Pechini. US Patent. 3330697 (1967).

[13] W. Kraus, G. Nolze. J. Appl. Cryst. 9, 301 (1996).

[14] Я. Смит, Х. Вейн. Ферриты. Иностр. лит., М. (1962). 504 с.

[15] D.D. Khalyavin, D.N. Argyriou, U. Amann, A.A. Yaremchenko, V.V. Kharton. Phys. Rev. B 75, 134407 (2007).

[16] T. Sarkar, V. Pralong, B. Raveau. Phys. Rev. B 83, 214428 (2011).

[17] B. Raveau, Ch. Simon, V. Caignaert, V. Pralong, F.X. Lefevre. J. Phys.: Condens. Matter 18, 10237 (2006).

[18] A.K. Kundu, V. Pralong, B. Raveau, V. Caignaert. J. Mater. Sci. 46, 681 (2011).

[19] C. Martin, A. Maignan, D. Pelloquin, N. Nguyen, B. Raveau. Appl. Phys. Lett. 71, 1421 (1997).

[20] M. Respaud, C. Frontera, J.L. García-Muñoz, M.Á.G. Aranda, B. Raquet, J.M. Broto, H. Rakoto, M. Goiran, A. Llobet, J. Rodríguez-Carvajal. Phys. Rev. B 64, 214401 (2001).

[21] E.P. Amaladass, N. Thirumurugan, A.T. Satya, C.S. Sundar, A. Bharathi. J. Phys. Condens. Mater., 25, 436001 (2013).

[22] C. Frontera, J.L. Garčia-Mun̆oz, A. Llobet, M.A.G. Aranda. Phys. Rev. B 65, 180405 (2002).

[23] G. Aurelio, J. Curiale, R.D. Sánchez, G.J. Cuello. Physica B 398, 223 (2007).

[24] С.В. Телегин, С.В. Наумов, О.Г. Резницких, Е.И. Патраков. ФTT 57, 2222 (2015).

[25] J.H. Van Vleck. The theory of electric and magnetic susceptibilities, University Press, Oxford (1952). 384 p.

[26] Р. Карлинг. Магнетохимия. Мир, М. (1989). 333 с.

[27] Y. Moritomo, T. Akimoto, M. Takeo, A. Machida, E. Nishibori, M. Takata, M. Sakata, K. Ohoyama, A. Nakamura. Phys. Rev. B 61, R 13325 (2000).

[28] P.M. Raccah, J.B. Goodenough. Phys. Rev. 155, 932 (1967).

[29] J.-P. Doumerc, J.-C. Grenier, P. Hagenmuller, M. Pouchard, A. Villesuzanne. J. Solid State Chem. 147, 211 (1999). 